\title{
Thrombocyte indices and plateletcrit in dogs with primary immune-mediated hemolytic anemia
}

\author{
EKREM ÇAĞATAY ÇOLAKOĞLU, ALI EVREN HAYDARDEDEOĞLU*
}

\begin{abstract}
Ankara University Faculty of Veterinary Medicine, Department of Internal Medicine, Ankara, Turkey
*Aksaray University Faculty of Veterinary Medicine, Department of Internal Medicine, Aksaray, Turkey
\end{abstract}

Çağatay Çolakoğlu E., Evren Haydardedeoğlu A.

Thrombocyte indices and plateletcrit in dogs with primary immune-mediated hemolytic anemia

Summary

Primary immune-mediated hemolytic anemia (IMHA) is one of the most common autoimmune disorders in dogs. Platelet indices in dogs with IMHA have not been commonly used in daily clinical practice. The purpose of this study was to evaluate trombocyte indices, including platelet count (PLT), mean platelet volume (MPW), platelet size deviation width (PDW), and plateletcrit (PCT) in dogs with primary IMHA and to compare them with the corresponding values in a control series of healthy dogs. This study was performed on 21 dogs diagnosed with primary IMHA and 11 healthy dogs. The inclusion criteria were the presence of a positive Coombs' test, true agglutination, anemia with HCT $<35 \%$, hyperbilirubinemia, bilirubinuria, hemoglobinemia, hemoglobinuria, and spherocytosis. MPV and PDW were significantly higher in the dogs with IMHA compared to the reference population. No significant differences were found in PLT and PCT between the groups. The use of MPV and PDW values in evaluating the bone marrow response would be helpful in dogs with primary IMHA.

Keywords: anemia, canine, hemolysis, platelet, plateletcrit

Primary immune-mediated hemolytic anemia (IMHA), characterized by destruction of erytrocytes, is one of the most common autoimmune disorders with no identifiable causative agent in dogs $(6,13)$. It has been reported that approximately $50-70 \%$ of dogs with IMHA had concurrent thrombocytopenia-induced bleeding complications $(10,24)$. Platelets play an important role in hemostasis and bleeding sites associated with vascular injury and autoimmune processes. Damaged endothelium, interactions with coagulation factors or autoimmune mechanisms induce platelet activation, aggregation and formation (8). Development of disseminated intravascular coagulopathy and changes in endothelial integrity and blood flow contribute to thrombus formation, creating the risk of death in dogs with IMHA (19).

Increased availability of automated blood analyzers has provided new approaches in evaluating platelet mass (25). Platelet indices, such as MPV and PDW, reveal changes in platelet size associated with an increased number of megakaryocytes in the bone marrow of dogs (22). Increased numbers of larger platelets also increase platelet heterogeneity (7). PCT is also a more sensitive indicator of platelet mass compared to platelet count (PLT) in dogs (18).

Although the potential usefulness of these platelet indices in dogs with IMHA has been recognized (2), they have not been commonly used in daily clinical practice. However, the ability of non-invasive parameters in evaluating the bone marrow response would be helpful in IMHA dogs. The purpose of the current study was therefore to evaluate trombocyte indices, including PLT, MPW, PDW and PCT, in dogs with primary IMHA and to compare them with a control series of healthy dogs.

\section{Material and methods}

Study population. This study was performed on 21 nonpregnant client-owned dogs diagnosed with primary IMHA at the Veterinary Teaching Hospital of Ankara University. The inclusion criteria were the presence of a positive Coombs' test (10), true agglutination persisting after dilution in phosphatebuffered saline, anemia with HCT $<35 \%$ (10), hyperbilirubinemia, bilirubinuria, hemoglobinemia, hemoglobinuria and spherocytosis (10). All dogs received routine vaccination against rabies, distemper, parainfluenza, hepatitis, parvoviral enteritis and leptospirosis. Dogs were not included in the study if they had been exposed to ehrlichiosis, borreliosis, anaplasmosis and dirofilariasis detected by the Idexx 4Dx Elisa antibody snap test. The study also did not include dogs receiving drug therapy, as well as dogs with $<30,000$ platelets/ $\mu 1(17)$, hemorrhage secondary to trauma, liver lobe torsion, impaired renal function, and evidence of toxication or neoplasia, as demonstrated by clinical procedures, such as physical examination, blood analyses (including serum profiles), imaging, urinalysis, cytology, or biopsy. The animals were treated with appropriate medications. Written consent was obtained from the owners.

Study design. All clinical procedures in dogs, including clinical examination, blood analyses, urinalysis, peripheral 
smears, and imaging (radiography and ultrasonography), were performed at the Veterinary Teaching Hospital. In addition to conventional hematological and biochemical parameters, platelet indices, including PLT, PCT, MPV, and PDW, were determined in all dogs at admission (Exigo Eos Vet, Hematology Analyzer, Erba XL Biochemical analyzer). The reference population consisted of 11 healthy dogs (excluding Cavalier King Charles Spaniels). The reference population had received the same vaccinations as the dogs with primary IMHA. Ehrlichiosis, borreliosis, anaplasmosis and dirofilariasis were also ruled out in the reference population by the Idexx 4Dx Elisa antibody snap test. Data were analyzed using the independent samples t-test. The level of $\mathrm{P}<0.05$ was considered as significant for statistical evaluation. Commercial statistical software was used for data analyses (SPSS version 23.0, Inc, USA).

\section{Results and discussion}

The group of twenty-one dogs with primary IMHA consisted of mixed breeds $(n=7)$, Cocker Spaniels $(\mathrm{n}=4)$, Golden Retrievers $(\mathrm{n}=2)$, Labrador Retrievers $(\mathrm{n}=2)$, a Pincher $(\mathrm{n}=1)$, a Kangal $(\mathrm{n}=1)$, a Bichon Frise $(n=1)$, a Pekingese $(n=1)$, a German Shepherd $(n=1)$, and a Pointer $(n=1)$. Seven dogs were excluded from the study because of a concurrent drug therapy, neoplasia, and renal and hepatic disease. The reference population consisted of 11 healthy dogs, including Terriers $(n=3)$, Cocker Spaniels $(n=2)$, Labrador Retrievers $(n=2)$, mixed breeds $(n=2)$, a Golden Retriever $(n=1)$, and a Pekingese $(n=1)$.

The mean age, weight and sex distributions of dogs in the groups are shown in Table 1 . The most common initial clinical signs in dogs with primary IMHA were weakness $(n=21)$, anorexia $(n=16)$, and icterus $(n=8)$. Tachycardia $(>120 \mathrm{bpm})$ was observed in the IMHA dogs. Splenomegaly and concurrent hepatomegaly were also the most common ultrasonographic finding in those dogs $(n=11)$. MPV and PDW were significantly higher in the IMHA dogs compared to the reference population. No significant differences in PLT or PCT were found between the groups (Tab. 2).

Primary IMHA is one of the most common autoimmune disorders with no identifiable causative agent in $\operatorname{dogs}(6,13)$. It is also a rule-out diagnosis among some other causes of secondary IMHA, such as rickettsial infections, toxin and drug exposure, neoplasia or hypophosphatemia $(1,4)$. In this study, we ruled out the other causes of secondary IMHA by anamnesis, physical examination, blood analyses (including serum profiles), imaging, urinalysis, cytology, biopsy, and snap tests. We could not compare the results of studies with larger cases concerning secondary thrombocytopenia (21). A predisposition to IMHA has been reported in Cocker Spaniels, Poodles, Setters, Bichons Frises, Pinschers and Collies $(4,10,19)$. In addition, clinical signs of anorexia, lethargy, weakness, dyspnea, and icterus have been reported in IMHA dogs $(1,10)$. In the present study, we
Tab. 2. Complete blood counts in the groups

\begin{tabular}{|c|c|c|c|}
\hline Variables & IMHA dogs & Reference population & $\mathbf{P}$ \\
\hline WBC $\left(10^{9} / l\right)$ & $14.46 \pm 2.92$ & $12.59 \pm 1.18$ & 0.65 \\
\hline LYM $\left(10^{9} / I\right)$ & $1.88 \pm 0.33$ & $3.15 \pm 0.57$ & 0.048 \\
\hline MON $\left(10^{9} / 1\right)$ & $0.93 \pm 0.20$ & $0.64 \pm 0.12$ & 0.34 \\
\hline NEUT $\left(10^{9} / 1\right)$ & $11.56 \pm 2.49$ & $8.80 \pm 0.90$ & 0.44 \\
\hline LYM (\%) & $15.57 \pm 2.46$ & $24.90 \pm 2.85$ & 0.026 \\
\hline MON (\%) & $8.52 \pm 1.41$ & $4.88 \pm 0.69$ & 0.082 \\
\hline NEUT (\%) & $75.23 \pm 3.29$ & $70.22 \pm 3.08$ & 0.33 \\
\hline $\operatorname{RBC}\left(10^{12} /\right)$ & $2.94 \pm 0.32$ & $6.79 \pm 0.41$ & 0.001 \\
\hline$H G B(g / d l)$ & $8.45 \pm 1.89$ & $13.57 \pm 0.81$ & 0.067 \\
\hline HCT (\%) & $19.32 \pm 1.86$ & $42.77 \pm 2.64$ & 0.001 \\
\hline $\operatorname{MCV}(f l)$ & $67.37 \pm 2.78$ & $64.03 \pm 2.51$ & 0.44 \\
\hline MCH (pg) & $22.03 \pm 0.98$ & $20.21 \pm 0.79$ & 0.23 \\
\hline MCHC (g/dl) & $32.11 \pm 0.77$ & $31.54 \pm 0.27$ & 0.61 \\
\hline RDW (\%) & $19.39 \pm 0.61$ & $15.00 \pm 0.38$ & 0.001 \\
\hline PLT $\left(10^{9} / \mathrm{I}\right)$ & $265.33 \pm 67.08$ & $290.27 \pm 37.31$ & 0.80 \\
\hline РСТ (\%) & $0.30 \pm 0.07$ & $0.24 \pm 0.03$ & 0.53 \\
\hline MPV (fl) & $12.19 \pm 0.81$ & $8.34 \pm 0.20$ & 0.002 \\
\hline PDW (\%) & $32.65 \pm 2.14$ & $15.91 \pm 0.09$ & 0.001 \\
\hline
\end{tabular}

Explanations: WBC - White blood cell; LYM - Lymphocyte; MON - Monocyte; NEUT - Neutrophil; RBC - Red blood cell; HGB - Hemoglobin; HCT - Hematocrit; MCV - Mean corpuscular volume; $\mathrm{MCH}$ - Mean corpuscular hemoglobin; MCHC - Mean corpus cular hemoglobin concentration; RDW - Red blood cell distribution width; PLT - Platelet; PCT - Plateletcrit; MPV - Mean platelet volume; PDW - Platelet distribution width

observed similar breeds and clinical signs consistent with the reports previously described. Contents resulting from premature destruction of RBCs by immune response have been accumulated in the spleen and liver in the body. Therefore, splenomegaly and concurrent hepatomegaly have been observed as the natural result of this process (1). In this study, splenomegaly and hepatomegaly were the most common ultrasonographic findings in IMHA dogs.

Platelets play an important role in bleeding sites and hemostasis. Damaged endothelium, interactions with coagulation factors, and autoimmune processes induce platelet activation, aggregation, and formation (8). Changes in endothelial integrity and blood flow contribute to thrombus formation, creating the risk of death in dogs with IMHA (19). Some dogs with IMHA have concurrent thrombocytopenia-induced bleeding complications, known as Evans syndrome $(10,24)$. In a study performed on 30 dogs (17), a cut-off platelet 
count for the diagnosis of immune-mediated thrombocytopenia with concurrent bleeding complications was set at 30,000 platelets/ $\mu 1$. However, the development of bleeding in thrombocytopenic dogs has been reported as unpredictable, and survival did not correlate with PLT (15). Platelet destruction and concurrent impaired platelet function could also be present in IMHA dogs (11). Although it was not the aim of this study to evaluate the correlation between platelet count and platelet function, we did not observe any signs of bleeding complications in dogs enrolled in the study. This is probably explained by the inclusion criterium $>30,000$ platelets/ $\mu$ l adopted in this study.

Values of MPV and PDW reveal changes in platelet size associated with increased number of larger platelets (megakaryocytes) (22). Larger platelets have been observed in blood when bone marrow was overstimulated to produce more platelets $(2,7)$. Blood levels of MPV and PDW also show an increased response to excessive platelet demand in IMHA dogs with concurrent thrombocytopenia $(2,12)$. Increased numbers of larger platelets also increase platelet heterogeneity (PDW) (7). In the current study, MPV and PDW, as indicators of immunemediated platelet consumption and heterogeneity, were significantly higher in IMHA dogs. Compared to MPV, PDW has been reported as a more sensitive and more reliable indicator of increased macroplatelets in distinguishing hyperdestructive thrombocytopenia from a hypoproductive one $(2,7)$. In this study, increased levels of PDW also reflect a regenerative hyperdestructive process. In two previous studies, increased MPV values were reported in dogs with regenerative thrombocytopenia (14, 22). However, some dogs with thrombocytopenia in those studies had lower MPV values. It was not consistent with the results obtained in this study. We suppose that lower MPV in some dogs in the previous studies was related to the severity of the immune response, inadequate bone marrow support, or the time frame in which the owners brought their dogs to the hospital.

The efficiency of platelet formation is related more closely to platelet mass than to platelet number (5). PCT is also a more sensitive indicator of platelet mass compared to PLT in dogs (18). In human emergency units, the use of PCT in making clinical decisions about transfusions has reduced the incidence of bleeding complications in transfusions (5). Although no statistically significant results were obtained in the study, dogs with primary IMHA had a larger PCT than the reference population, similarly to the report previously described (20).

In a study of Cavalier breed dogs with genetic macrothrombocytopenia related to the autosomal recessive dysplasia of platelets, PCT was demonstrated to be numerically incorrect in assessing platelet mass $(9,16$, $23)$. For this reason, Cavalier King Charles Spaniels were not included in the current study. The lack of research for hereditary disorders, such as pyruvat kinase and phosphofructokinase deficiencies, in canine breeds was a limitation of the current study.

In conclusion, we have demonstrated that, although no statistically significant differences in PCT were observed between the groups, MPV, PDW, and PCT values were higher in dogs with primary IMHA compared to the reference population. The use of MPV and PDW values in evaluating the bone marrow response would be helpful in dogs with primary IMHA.

\section{References}

1. Balch A., Mackini A.: Canine immune-mediated hemolytic anemia: pathophysiology, clinical signs, and diagnosis, compendium on continuing education for the practising veterinarian. Compendium 2007, 29, 217-225.

2. Bommer N. X., Shaw D. J., Milne E. M., Ridyard A. E.: Platelet distribution width and mean platelet volume in the interpretation of thrombocytopenia in dogs. JSAP 2008, 49, 518-524.

3. Botsch V., Küchenhoff H., Hartmann K., Hirschberger J.: Retrospective study of 871 dogs with thrombocytopenia. Vet. Rec. 2009, 164, 647-651.

4. Carr A. P., Panciera D. L., Kidd L.: Prognostic factors for mortality and thromboembolism in canine immune-mediated hemolytic anemia: A retrospective study of 72 cases. JVIM 2002, 16, 504-509.

5. Gerday E., Baer V. L., Lambert D. K., Paul D. A., Sola-Visner M. C., Pysher T. J., Christensei R. D.: Testing platelet mass versus platelet count to guide platelet transfusions in the neonatal intensive care unit. Transfusion 2009, 49, 2034-2039.

6. Jain N. C. (ed.): Hemolytic anemia of noninfectious origin, [in:] Schalm's Veterinary Hematology. Lea\&Febiger, Philadelphia 1986, p. 647-651.

7. Kaito K., Otsubo H., Usui N., Yoshida M., Tanno J., Kurihara E., Matsumoto K., Hirata R., Domitsu K., Kobayashi M.: Platelet size deviation width, platelet large cell ratio, and mean platelet volume have sufficient sensitivity and specificity in the diagnosis of immune thrombocytopenia. BJH 2005, 128, 698-702.

8. Kamath S.: Platelet activation: assessment and quantification. Europ. Heart J. 2001, 22, 1561-1571.

9. Kelley J., Sharkey L. C., Christopherson P., Rendahl A.: Platelet count and plateletcrit in Cavalier King Charles Spaniels and Greyhounds using the advia 120/2120. Vet. Clin. Pathol. 2014, 43, 43-49.

10. Klag A., Giger U., Shofer F.: Idiopathic immune-mediated hemolytic anemia in dogs: 42 cases (1986-1990). JAVMA 1993, 202, 783-788

11. Kristensen A. T., Weiss D. J., Klausner J. S.: Platelet dysfunction associated with immune-mediated thrombocytopenia in dogs. JVIM 1994, 8, 323-327.

12. Loo B., Martin J. F.: Megakaryocytes and platelets in vascular disease. Baillieres Clin. Haematol. 1997, 10, 109-123.

13. McCullough S.: Immune-mediated hemolytic anemia: understanding the nemesis Vet. Clin. North Am. Small Anim. Pract. 2003, 33, 1295-1315.

14. Northern J., Tvedten $H$. W.: Diagnosis of microthrombocytopenia and immunemediated thrombocytopenia in dogs with thrombocytopenia: 68 cases (1987-1989). JAVMA 1992, 200, 368-372.

15. O'Marra S. K., Delaforcase A. M., Shaw S. P.: Treatment and predictors of outcome in dogs with immune-mediated thrombocytopenia. JAVMA 2011, 238, 346-352

16. Pedersen H. D., Haggström J., Olsen L. H., Christenseni K., Selin A., Burmeister M. L., Larsen H.: Idiopathic asymptomatic thrombocytopenia in Cavalier King Charles Spaniels is an autosomal recessive trait. JVIM 2002, 16, 169-173.

17. Putsche J. C., Kohn B.: Primary immune-mediated thrombocytopenia in 30 dogs (1997-2003). JAAHA 2008, 44, 250-257.

18. Schwartz D., Sharkey L., Armstrong P. J., Knudson C., Kelley J.: Platelet Volume and Plateletcrit in Dogs with Presumed Primary Immune-Mediated Thrombocytopenia. JVIM 2014, 28, 1575-1579.

19. Scott-Moncrieff J. C., Treadwell N. G., McCullough S. M., Brooks M. B. Hemostatic abnormalities in dogs with primary immune-mediated hemolytic anemia. JAAHA 2001, 37, 220-227.

20. Smith J. R., Smith K. F., Brainard B. M.: Platelet parameters from an automated hematology analyzer in dogs with inflammatory clinical diseases. Vet. J. 2014, 201, 406-411.

21. Souza A. M., Pereira J. J., Campos S. D. E., Filho R. A., Xavier M. S., Bacellar D. T. L., Almosny N. R. P.: Platelet indices in dogs with thrombocytopenia and dogs with normal platelet counts. Arch. Med. Vet. 2016, 48, 277-281.

22. Sullivan P. S., Manning K. L., Mcdonald T. P.: Association of mean platelet volume and bone marrow megakaryopoiesis in thrombocytopenic dogs: 60 cases (1984-1993). JAAHA 1995, 206, 332-334

23. Tvedten H., Lilliehöök I., Hillström A., Häggström J.: Plateletcrit is superior to platelet count for assessing platelet status in Cavalier King Charles Spaniels. Vet. Clin. Pathol. 2008, 37, 266-271.

24. Weiss D. J., Brazzell J. L.: Detection of activated platelets in dogs with primary immune-mediated hemolytic anemia. JVIM 2006, 20, 682-686.

25. Žvorc Z., Bari Rafaj R., Kuleš J., Mrljak V.: Erythrocyte and platelet indices in babesiosis of dogs. Vet. Arhiv. 2010, 80, 259-267.

Corresponding author: Dr. Ekrem Cağatay Colakoğlu, Ankara University Faculty of Veterinary Medicine, Turkey; e-mail: colakoglu@ankara.edu.tr 